\title{
Assessment of aflatoxins, ochratoxin A and zearalenone in breakfast cereals
}

\begin{abstract}
Aflatoxins (AFs), ochratoxin A (OTA) and zearalenone (ZEN) were analysed in 237 breakfast cereal samples collected from central areas of Punjab, Pakistan. According to the results, $41 \%$ of the samples were found contaminated with AFs, out of which $16 \%$ and $8 \%$ samples were found to be above the European Union (EU) maximum content for AFB1 and total AFs, respectively. About $48 \%$ samples were found contaminated with OTA and $30 \%$ samples were found to be above the EU maximum content. The results have shown that 53\% samples of breakfast cereals were found contaminated with ZEN and $8 \%$ samples were found to be above the permissible limit of EU. The highest mean level of AFB1 and total AFs were found in semolina i.e. 3.60 and $4.55 \mu \mathrm{g} / \mathrm{kg}$, respectively. Similarly, semolina was the highest contaminated breakfast cereal for OTA $(3.90 \mu \mathrm{g} / \mathrm{kg})$, while cornflakes (brand B) was found highest contaminated with ZEN $(13.45 \mu \mathrm{g} / \mathrm{kg})$.
\end{abstract}

Keyword: Mycotoxins; Aflatoxins; Ochratoxin A; Zearalenone; Breakfast cereals 